Revue

Revue de l'histoire des religions

de Ihistoire des religions

\title{
Le potentiel des peaux d'animaux sacrifiés dans le monde gréco-romain
}

The Potential of Sacrificial Animal Skins in the Greco-Roman World

\section{Robert Turcan}

\section{OpenEdition}

\section{Journals}

Édition électronique

URL : https://journals.openedition.org/rhr/9877

DOI : $10.4000 /$ rhr.9877

ISSN : 2105-2573

\section{Éditeur}

Armand Colin

\section{Édition imprimée}

Date de publication : 1 septembre 2019

Pagination : 459-473

ISBN : 978-2-200-93260-2

ISSN : 0035-1423

Référence électronique

Robert Turcan, «Le potentiel des peaux d'animaux sacrifiés dans le monde gréco-romain », Revue de I'histoire des religions [En ligne], 3 | 2019, mis en ligne le 01 janvier 2022, consulté le 08 janvier 2022. URL : http://journals.openedition.org/rhr/9877 ; DOI : https://doi.org/10.4000/rhr.9877 


\section{Le potentiel des peaux d'animaux sacrifiés dans le monde gréco-romain}

C'est une tradition antique de conserver la peau des victimes sacrificielles, censée détenir une part de vertu divine. La dépouille servait aux cérémonies de purification (I), à la divination (II) ou à l'identification rituelle du fidèle avec son dieu (III). Les procédures concernant l'avenir à prévoir, en liaison avec l'incubation, sont rappelées et analysées, compte tenu de leurs variantes suivant les contextes.

\section{The Potential of Sacrificial Animal Skins in the Greco-Roman World}

It was a tradition in ancient times to keep the skins of sacrificial animals, which were supposed to contain a portion of divine virtue. Such remains were used for purification ceremonies (I), for divination (II) or for a worshiper's ritual identification with his god (III). The procedures involved in foretelling the future, in connection with incubation rituals, are recalled and analyzed, along with their variations according to different contexts.

* N.D.L.R. : Le Professeur Robert Turcan est décédé le 16 janvier 2018. Il nous avait adressé le 12 janvier son texte que la Revue s'honore de publier et dont la mise au point a été confiée à notre collègue Sylvain Destephen (Université Paris Nanterre), membre du comité de rédaction. 
Grâce au contact avec une partie de la victime sacrifiée, on se réapproprie, en somme, quelque chose du potentiel divin acquis par cette victime, car en la sacralisant, comme l'indique le nom latin du sacrifice (sacrificium), on la « soustrait à l'usage profane $»^{1}$. L'animal sacrifié «est entré dans la zone du divin $»^{2}$. C. Trebatius Testa écrivait dans le premier livre de son De religionibus qu'est «sacré tout ce que l'on tient pour être la propriété des dieux $»^{3}$. On a reconnu depuis longtemps que «le sacrifice... est le lien essentiel entre l'homme et la divinité $»^{4}$. Comme l'affirmait Platon, « sacrifier aux dieux, c'est entrer en relation avec eux $»^{5}$.

Les chrétiens en étaient tout aussi convaincus, car ils croyaient en l'existence des dieux en tant que «démons », et c'est pour cette raison qu'ils refusaient de consommer la viande des animaux sacrifiés, qu'on trouvait plutôt à meilleur compte sur les marchés. Pour eux, il s'agissait, en effet, de chairs dont la consécration aux démons contaminait la substance et qu'infectait dès lors une souillure maléfique. Tout ce qui est devenu la propriété des dieux, en porte l'empreinte.

C'est singulièrement le cas pour les peaux d'animaux immolés suivant les règles du rituel. Dans l'univers magico-religieux de l'Antiquité classique, comme dans le folklore de l'humanité en général, s'affirme ou du moins affleure une croyance aux vertus de certaines dépouilles animales et plus précisément de victimes sacrales. Les procédures connues du contact de ces dépouilles coïncident, moyennant des variantes, mais avec une même référence à leur pouvoir surnaturel, avec trois fonctions différentes : purification, divination, déification.

1. Jean Bayet, Histoire politique et psychologique de la religion romaine ${ }^{2}$, Paris, Payot, 1969 («Bibliothèque historique »), p. 129.

2. Ibid., p. 131.

3. Macr., Sat., 3, 2 : sacrum est quicquid est quod deorum habetur. Ami intime de Cicéron, Trebatius Testa «s'est consacré surtout au droit». Cf. Henry Bardon, La littérature latine inconnue, 1. L'époque républicaine ${ }^{2}$, Paris, Klincksieck, 2014 ( Série Littérature », 6), p. 308.

4. Salomon Reinach, Cultes, mythes et religions, I3, Paris, Leroux, 1922, p. 97.

5. Plat., Leg., IV, 716 d. 


\section{I/ LA PURIFICATION}

Il convient d'être pur pour faire acte de piété : «Pur doit être celui qui pénètre dans un temple où brûle l'encens, et la pureté consiste à penser saintement», pouvait-on lire à l'entrée du sanctuaire d'Épidaure ${ }^{6}$. Donc, on ne peut procéder à un rite lustral qu'à condition d'être pur moralement et religieusement, qu'on soit fidèle ou prêtre.

Cette exigence apparait dans la forme la plus archaïque et la plus solennelle du mariage romain : la confarreatio, à laquelle procède le flamine de Jupiter, lui-même marié suivant le même rituel. Après le sacrifice d'une brebis, sa toison est étalée sur deux sièges conjoints qu'occupent les futurs époux ${ }^{7}$. Le contact avec une dépouille affiliée désormais à l'essence divine les met, pour ainsi dire, dans un état de grâce propice au partage du fameux gâteau d'épeautre qui consacrera leur union. Or, « parmi les rites religieux, il n'y avait pas de plus sacré que le mariage par confarréation $»^{8}$.

Un parallèle s'impose avec le passage des Argonautiques où Apollonius de Rhodes ${ }^{9}$ évoque l'antre où Jason et Médée se sont unis sur la peau $d u$ bélier ailé, cette toison d'or que Phrixos avait consacrée à Mars ${ }^{10}$, « dans un bois sacré d'Arès ${ }^{11}$, gardée (pendue à un chêne) par un dragon qui ne dormait jamais. Sans doute informé là par une donnée ancienne de l'érudition alexandrine, Apollonius en a méconnu la signification religieuse, car il écrit tout simplement que la toison servait à honorer un mariage « digne d'être chanté » ${ }^{12}$.

6. Clem. Alex., Strom., V, 1, 13, 3 ; Porph., Abst., II, 19, 5 ; Cyr. Alex., C. Iul., IX, 310 c-e, dans PG, 76, col. 977 B-C. Cf. Cic., Leg., II, 8, 24 (Caste iubet lex adire ad deos, animo uidelicet, quo sunt omnia); ND, II, 28, 71 (cultus autem deorum optimus... ut eos semper pura, integri incorrupta et mente et ueneremur). Cette tradition remonte à Théophraste et à son traité de la piété : Eugen Fehrle, Die kultische Keuschheit im Altertum, Giessen, Töpelmann, 1910 («Religionsgeschichtliche Versuche und Vorarbeiten », 6 [désormais RGVV]), p. 49 ss., 69 ss.

7. Fest., su, In pelle lanata, 102, 1-3 Lindsay (noua nupta consedere solet...); Deutero-Seru., Aen., IV, 374 (I, p. 530, 30-531,1-2 Thilo) : sellas duas iugatas ouilla pelle superiniecta.

8. Plin., NH, 18, 10. Cf. Georges Dumézil, Mariages indo-européens, Paris, Payot, 1979 («Bibliothèque historique »), p. 48.

9. Arg., IV, 1141-1155.

10. Hyg., Fab., XII, 3 ; XXII, 1.

11. Apollod., Bibl., I, 9, 16, 107.

12. Arg., IV, 1142-1143. 
Le flamine de Jupiter, qui préside au mariage par confarreatio, doit porter, comme en d'autres cérémonies, un bonnet taillé dans la peau d'un taureau blanc immolé à son dieu ${ }^{13}$.

Pour implorer les dieux, il importe parfois de prendre l'aspect de la victime sacrificielle. À Magnésie du Pélion, dans une procession pour la pluie ${ }^{14}$, les premiers citoyens de la ville, choisis par le prêtre, montent jusqu'à l'antre de Chiron et au temple de Zeus Akraios (ou plutôt Aktaios, comme nous en instruit l'épigraphie), revêtus des peaux de béliers immolés préalablement, d'après le Pseudo-Dicéarque de Messène ${ }^{15}$.

Sur un sarcophage (ou ostéothèque ?) de Torre Nova (Rome, Palais Borghèse), comme sur l'urne «Lovatelli » et des plaques Campana ${ }^{16}$, un Héraclès voilé, un pied sur la dépouille d'un bélier (Dios kôdion ou Dion kôdion), animal consacré à Zeus Meilichios ou Ktésios $"$ pour des purifications $»^{17}$. La Souda écrit «aux pieds des maudits», Hesychius parle du «pied gauche». Sur le sarcophage, il s'agit du pied droit. Ce Dion Kôdion était censé absorber toutes les impuretés qu'il convenait d'expulser

13. Gell. NA, X, 15, 32 (Is solum album habet galerum, uel quod maximus, uel quod Ioui immolata hostia alba fieri oporteat) ; Deutero-Seru., Aen., II, 683 (I, p. 318, 25-26), d'après Suet., De genere uestium, fr. 168, p. 268 Reifferscheid $^{2}$, Leipzig, 1963. Cf. Front., Ep., IV, 4, 1 (p. 60, 12 Van Den Hout) ; Samson Eitrem, Opferritus und Voropfer der Griechen und Römer ${ }^{2}$. Hildesheim-New York, Olms, 1977, p. 384 ; Jens H. Vanggaard, The Flamen. A Study in the History and Sociology of Roman Religion, Copenhague, Museum Tusculanum Press, 1988, p. 40 et 160 .

14. Arthur Bernard Cook, Zeus. A Study in Ancient Religion, 1, Cambridge, Cambridge University Press, 1914, p. 164-165, 420 ss. ; 2, Cambridge, 1916, p. 869 , n. 2 ; S. Eitrem, Opferritus, p. 372.

15. FHG, II, p. 262, 8, fr.60. Cf. James George Frazer, Sur les traces de Pausanias. À travers la Grèce ancienne, trad. franç. ${ }^{2}$, Paris, Les Belles Lettres, 1927, p. 61-62 (où est justement soulignée la « signification religieuse » de la cérémonie); Ludwig Preller, Griechische Mythologie, 1. Theogonie, Berlin, Weidmann, 1894, p. 144 et n. 1 ; rapprochement du Dios kôdion, pour la vertu que détient la peau d'un bélier sacrifié.

16. Giulio Emanuele Rizzo, «Il sarcofago di Torre Nova», Römische Mitteilungen, t. 25, 1910, p. 89-167 ; George Emmanuel Mylonas, Eleusis and the Eleusinian Mysteries, Princeton, Princeton University Press, 1961, fig. 83-84 ; Ugo Bianchi, The Greek Mysteries, Leyde, Brill, 1976 («Iconography of Religions », 18, 3), p. 9 et 27-29, ill. n ${ }^{\text {os }}$ 47-52 ; Walter Burkert, Ancient Mystery Cults, Cambridge (Mass.) - Londres, Harvard University Press, 1987, p. 94 s. («purification scene »), fig. 2-4.

17. Hesych., $s u$; Soud., su ; Eustath, Od., 1935, 8, probablement d'après Polémon d'Ilion : Aloysius Tresp, Die Fragmente der griechischen Kultschriftsteller, Giessen, Töpelmann, 1914 («RGVV», 15, 1), p. 86-87, nos 43-44. 
des purifications ${ }^{18}$. Comme les Petits Mystères d'Éleusis, qu'on célébrait au printemps (saison des purifications) passaient pour avoir été créés par Déméter pour purifier Héraclès du meurtre des Centaures ${ }^{19}$, et comme il était étranger, c'est aussi pour lui ${ }^{20}$ qu'on $^{\text {' }}$ avait institué cette « initiation préalable $»^{21}$.

En dehors même des cultes initiatiques, certains prêtres et parfois des fidèles doivent revêtir une peau d'animal sacrifié, comme on l'a vu pour le flamine de Jupiter et dans la procession du Pélion.

On attachait des aétites aux femmes enceintes ou aux femelles pleines au moyen de peaux d'animaux immolés rituellement, pelliculis sacrificatorum animalium ${ }^{22}$, ce qui passait pour maintenir en place les enfants à naître, faute de quoi il y avait ptose de la vulve. On les enlevait au moment de l'accouchement, sinon la mise au monde était impossible! Dans les Argonautiques de Valerius Flaccus, les peaux des brebis noires sacrifiées aux dieux infernaux sont en partie portées dans les rangs de l'armée ${ }^{23}$.

Quand on se rend pour la première fois dans la ville sacrée d'Atargatis, au temple d'Hiérapolis-Bambykè, pour faire agréer un sacrifice à la déesse, on se rase la tête et les sourcils. Il faut immoler une brebis. On en découpe la peau qu'on étend par terre, on se met à genoux en mettant sur sa tête les pieds et le chef de l'animal (ce qui est une façon de s'identifier avec la victime) ${ }^{24}$.

18. Paul Stengel, Die griechischen Kultusaltertümer ${ }^{3}$, Berlin - Munich, C.H. Beck'sche Verlagsbuchhandlung, 1920 (« Handbuch der Klassischen Altertumswissenschaft », 5, 3), p. 164 ; Pierre Boyancé, «Un rite de purification dans les Argonautiques de Valerius Flaccus », Revue des études latines, t. 13, 1935, p. 128, repris dans Études sur la religion romaine, Rome, De Boccard, 1972 ( Collection de l'École française de Rome », 11), p. 337.

19. Diod. Sic., IV, 14, 3. Les Petits Mystères constituent comme une purification préliminaire, prokatharsis : Schol. Aristoph., Plut., 845. Sur Héraclès, cf. Maurice Brillant, Les mystères d'Éleusis, Paris, La Renaissance du Livre, 1920, p. 61 et 67.

20. Schol. Lycophr., Alex., 1328.

21. Pierre Roussel, «L'initiation préalable et le symbole éleusinien », Bulletin de Correspondance hellénique, t. 54, 1930, p. 62 ; W. Burkert, Ancient Mystery Cults, p. 90.

22. Plin., $N H, 36,151$.

23. Arg., III, 439-443. Cf. P. Boyancé, Études, p. 337-338, qui rapproche le Dios kôdion et les sacrifices offerts à Zeus Meilichios.

24. Lucian., D. Syr., 55. 
En Phénicie également s'imposait la croyance qu'on s'acquiert, par la peau des victimes, les vertus lustrales inhérentes à l'immolation $^{25}$. Cette force est communiquée en Égypte à l'idole du dieu Ammon, dont on la revêt, après le sacrifice du bélier ${ }^{26}$. On conservait la peau du bouc tué sur le champ même de la moisson, comme renfermant une partie de la vie divine ${ }^{27}$. On connaît le pouvoir défensif de l'égide sur la poitrine d'Athéna, ou d'Anat en Phénicie, comme de certains empereurs ${ }^{28}$. La léontè, sur la tête de ceux qui prétendaient réincarner la uirtus du héros de Némée, avait une signification similaire ${ }^{29}$.

En Italie, à Furfo (Santa Maria di Furfona), la lex Furfensis ${ }^{30}$ édicte que la peau des victimes sacrifiées à Jupiter Liber ou à son Genius reste la propriété exclusive du temple, en raison même de son potentiel sacré.

Le passage d'une année à l'autre donne lieu à des procédures cathartiques impliquant l'usage des peaux consacrées. L'année romaine commençait primitivement, comme on sait, en mars. Mais c'est le 14 qu'on priait Jupiter pour une année prospère. On lui sacrifiait un taureau de six ans pour les champs montagnards. C'est alors qu'une procession était conduite par un homme vêtu de dépouilles animales. On l'appelait Mamurius. Ce rituel consistait à expulser le «vieux mars $»^{31}$. Le lendemain, on fêtait Anna Perenna,

25. René Du Mesnil du Buisson, «L'ancien dieu tyrien », Mélanges de l'Université Saint-Joseph, t. 41, 1965, p. 8.

26. J. G. Frazer, Le rameau d'or, p. 471.

27. Ibid., p. 449.

28. Pour Anat, cf. René Du Mesnil du Buisson, Études sur les dieux phéniciens hérités par l'Empire romain, Leyde, Brill, 1970 («Études préliminaires sur les religions orientales dans l'empire romain », 14), p. 136 ; pour l'iconographie des empereurs, voir Andreas Alföldi, Die monarchische Repräsentation im römischen Kaiserreiche, Darmstadt, Wissenschaftliche Buchgesellschaft, 1970, p. 239-240 ; Robert Turcan, "Le culte impérial au $\mathrm{III}^{\mathrm{e}}$ siècle », Aufstieg und Niedergang der Römischen Welt, t. II, 16, 2, p. 1040-1041.

29. Ibid., p. 1041.

30. CIL, IX, $3513=I L S, 4906$. Cf. Robert E. A. Palmer, Roman Religion and Roman Empire. Five Essays, Philadelphie, University of Pennsylvania Press, 1974 (« The Harvey Foundation Series », 154), p. 59-60, 83, 136, 143.

31. Georges Dumézil, La religion romaine archaïque ${ }^{1}$, Paris, Payot, 1966 (réédité), p. 216-217 ; d'après Lyd., Mens., IV, 49 (p. 105, 19-106, 13 Wuensch). Cf. Seru., Aen., VII, 188 (II, p. 140, 24-25 Thilo), d'après qui Servius aurait écrit peltam feriunt, et non pellem uirgis feriunt. Le même problème se pose chez Minucius Felix (Oct., 24, 11), où l'on lit pelles caedunt, traduit par Jean Beaujeu (éd.-trad., Paris, Les Belles Lettres, 1964 («Collection des Universités 
époque intermédiaire et donc incertaine, avant mars qui ouvrait un recommencement, un ordre rétabli dans le cours des choses.

La tradition littéraire est unanime pour y reconnaître une fête de purifications ${ }^{32}$. On y fête Junon Februata, désignation ancienne de la cérémonie ${ }^{33}$. Or, nous explique Censorinus ${ }^{34}$, «februum, c'est tout ce qui expie et purifie. Les februamenta sont toutes les purifications. De même, februare signifie purger, purifier». Les femmes sont purifiées par la «peau de Junon», id est pelle caprina $^{35}$, la Junon de Lanuuium $^{36}$ qu'on représentait coiffée d'une peau de chèvre. Pour Ovide ${ }^{37}$, c'est la fête du dieu Faunus, qu'on identifiait avec le Pan d'Arcadie, porteur aussi de l'épiclèse Lukaios, autrement dit un «dieu-loup ».

Les femmes sont purifiées par la peau des chèvres immolées. On parle aussi d'un chien ou de chiots offerts à Hécate ${ }^{38}$. Dans la dépouille des chèvres, on découpe des lanières, qui serviront à frapper les femmes stériles pour les rendre fécondes. Ceints eux-mêmes de la peau des chèvres sacrifiées, les Luperques ainsi sacralisés font le tour du Palatinn ${ }^{39}$, à partir du Lupercal, refuge des jumeaux fondateurs :

de France »), p. 40 : «ils frappent des peaux de tambour », dans une phrase où il est question de «promener à la ronde de vieux boucliers », allusion possible à la procession de Mamurius. Mais le texte de Lydus est explicite.

32. DH, AR, I, 80, 1 ; Plut., Rom., 21, 4 ; Num., 19, 8 ; QR, 68, 280 b.

33. Plut., Rom., 21, 4. Cf. Thomas Köves-Zulauf, Römische Geburtsriten, Munich, C. H. Beck, 1990 («Zetemata », 87), p. 251-253, 281-284.

34. De die nat., 22, 14-16: Est februum quidquid expiat purgatque, et februamenta purgamenta, item februare et purum facere.

35. Fest., p. 75, 23 ss. - 76, 1 Lindsay.

36. Cf. Arthur Ernest Gordon, The Cults of Lanuvium, Berkeley, University of California Press, 1938 ( «University of California, Publications in Classical Archaeology », 2, 2), p. 32-33; Uberto Pestalozza, Religione Mediterranea. Vecchi e Nuovi Studi, Milan, Fratelli Bocca, 1951, p. 371-373.

37. F, II, 267-268, 271, 424. Il s'agit là d'une tradition savante, étrangère à l'année liturgique de Rome.

38. Plut., Rom., 21, 8.

39. Varr., LL, VI, 24 (lustratur antiquum oppidum Palatinum); DH, AR, I, 80, 1. Ces textes ont suscité un débat qui n'est pas clos. Cf. Adam Ziolkowski, "Le débat moderne sur le tracé de la course des Luperques », Revue des études latines, t. 94, 2016, p. 21-45. Ce «mythe de l'historiographie moderne » (ibid., p. 21 et 43) correspond à l'interprétation et aux traductions traditionnelles des deux textes précités. Tout dépend du préverbe peri- chez Denys d'Halicarnasse et Plutarque. Pour A. Ziolowski, il donne au verbe perielthein (chez Denys) et au nom peridromè (chez Plutarque), le sens d'aller ci et là, à travers la ville. Mais il s'agit du Palatin, et l'on ne voit pas précisément comment circulaient les Luperques par rapport au Palatin. Ils ont pu courir désordonnément, sans cesser de la faire autour de la colline. Seul nous importe ici l'usage des peaux de bêtes immolées. 
exemple typique d'une procédure qui consiste à faire le tour de l'être, de l'objet ou du site à purifier, mais que renforcent en l'occurrence les peaux animalières des victimes sacrificielles.

On amène aux Luperques deux jeunes garçons de famille noble. Après le sacrifice, certains membres de la confrérie leur touchent le front avec un couteau ensanglanté, d'autres les essuient aussitôt avec de la laine imbibée de lait ${ }^{40}$. Plutarque s'avoue incapable de comprendre les raisons de ce rituel, qui a bien tout l'air d'être un sacrifice humain fictif, le sacrifiant s'identifiant symboliquement, là comme ailleurs, avec le sacrifié.

Quoi qu'il en soit, les porteurs de la peau des victimes en acquéraient le pouvoir de purifier les femmes et la cité, dont l'avenir dépendait de leur fécondité.

Curieusement, Virgile évoquant le culte d'Hercule à l'Ara Maxima nous montre les prêtres et Potitius qui s'avancent « ceints de peaux suivant l'usage ${ }^{41}$. Le Deutéro-Servius explique ce détail surprenant en relation avec Pan, dieu arcadien équivalant à Faunus qui patronnait les Lupercales ${ }^{42}$. Évandre, venu d'Arcadie, passait pour avoir fondé le Lupercal ${ }^{43}$. Cependant, le culte d'Hercule à l'Ara Maxima est moins ancien que le croyait Virgile. Cet HerculeHéraclès, venu d'Italie méridionale, était un héros «chthonien et dionysiaque $»^{44}$. Je doute néanmoins qu'il ait été "porté par la faveur de... sectes dionysiaques $»^{45}$, plutôt que par les courants commerciaux du Forum Boarium ${ }^{46}$. Mais l'Ara Maxima avoisinait les pieds de l'Aventin où, en 186 av. J.-C., les Bacchanales feront du bruit, et où le temple, que Cérès partageait depuis 497 avec Liber et Libera, n'avait pu rester indifférent aux influences de la Grande Grèce ${ }^{47}$. Les peaux sur les reins des prêtres d'Hercule les mettent en état de célébrer le culte, comme des Luperques.

40. Ibid., 21, 6 .

41. Aen., VIII, 282 : pellibus in morem iuncti.

42. Ou., $F$, II, 423-424.

43. DH., $A R$, I, 32, 5 et 79, 8 ; Plut., Rom., 21, 4-5.

44. Jérome Carcopino, Aspects mystiques de la Rome païenne ${ }^{6}$, Paris, L'Artisan du livre, 1942, p. 191.

45. Ibid., p. 205.

46. Cf. Clara Gallini, Protesta e integrazione nella Roma antica, Bari, Laterza, 1970, p. 34-35.

47. Cf. Jean-Marie Pailler, Bacchanalia. La répression de 186 av. J.-C. à Rome et en Italie, Rome, De Boccard, 1988 (« Bibliothèque des Écoles françaises d'Athènes et de Rome », 270), p. 130 et passim. 


\section{II/ LA Divination}

L'homme est soucieux de l'avenir, qu'il soit immédiat ou lointain. Les dieux en détiennent le secret. Dans l'urgence, on ne va pas consulter les chênes de Dodone ou la pythie de l'Apollon delphique, car il faudrait recourir à des exégètes incertains, aux interprètes des énigmes oraculaires

La divination par les songes (onirocritique ou oniromancie) est universellement populaire dans l'Antiquité et l'est restée jusqu'à nos jours. Il s'agit d'une divination «intuitive », inspirée, dite aussi «naturelle », qu'on oppose à la divination «inductive » ou artificielle, qui repose sur l'observation de signes ou de présages inattendus. Or l'homme a tendance à préférer la première : «il fallait mettre l'âme directement en rapport avec les dieux ou les héros $»^{48}$. Mais l'oniromancie requérait néanmoins l'accomplissement de rituels particuliers, où les peaux de victimes sacrificielles jouaient un rôle essentiel. Il s'agit de rituels liés à l'incubation. Certes, «La Terre est mère des songes $»^{49}$. Mais pour avoir un rêve véridique, il convient de s'entendre avec un dieu ou un héros.

Dans les sanctuaires d'Asclépios, on s'endormait en principe non pas pour recevoir une prophétie, mais pour se faire dicter en rêve une ordonnance, que le médecin devait exécuter. Cependant, d'après saint Jérôme ${ }^{50}$, il fallait dormir sur les peaux d'animaux sacrifiés. Nous ne savons pas de quel temple il veut parler. Son information pourrait bien être entachée de confusion ou de généralisation abusive.

Mais, d'une façon générale, pour obtenir une prédiction, quand on demeure perplexe sur l'avenir, il faut se rendre dans un site oraculaire. Le devin homérique Calchas était héroïsé au

48. Auguste Bouché-Leclercq, Histoire de la divination dans l'Antiquité, 1, Paris, Leroux, 1879, rééd., Grenoble, Millon, 2003, p. 274. Cf. Raymond Bloch, La divination. Essai sur l'avenir et son imaginaire, Paris, Fayard, 1991, p. 38 : « vision directe d'un dieu ou de quelque être surnaturel».

49. Henri Lechat, dans Edmond Daremberg et Charles Saglio, Dictionnaire des antiquités grecques et romaines [désormais DAGL], III, 1, Paris, Hachette, 1900, rééd., Graz, Akademische Druck- und Verlagsanstalt, 1963, p. 458, s.u. Incubatio.

50. Comm. in Isaiam, 65, $4:$ in delubris idolorum... stratis pellibus hostiarum incubare soliti erant, ut somniis futura cognoscerent. Quod in fano Aesculapii usque hodie error celebrat. 
mont Drion en Daunie (Apulie), non loin de Podalirios. D'après Strabon $^{51}$, dans l'hérôon de Calchas, on ne pouvait consulter le prophète qu'à condition d'avoir immolé un bélier noir et de dormir sur sa peau. Un rite d'incubation était parallèlement observé dans l'hérôon de Podalirios, fils d'Asclépios, si l'on en croit deux vers de Lycophron $^{52}$ : «à tous ceux qui s'endormiront sur sa tombe, enveloppés de peaux de brebis, il dira en songe une parole véridique ». Il s'agit apparemment de brebis sacrifiées.

À Oropos en Béotie, l'oracle d'Amphiaraos avait d'autres exigences $^{53}$. Il fallait une purification préalable ${ }^{54}$. On devait renoncer au vin durant trois jours et jeûner une journée entière, avant de sacrifier un bélier et de se coucher sur la peau de la victime. Dans l'antre «sacré et divin », où méditait le devin, on voyait la Vérité en robe blanche, la porte des songes, le Rêve avec un visage où se peignait l'abandon, vêtu d'une robe blanche par dessus une noire, afin de signifier que le jour et la nuit lui appartiennent. Il avait entre les mains une corne indiquant qu'il introduit les songes par la porte de Vérités5. Mais c'est la peau du bélier sacrifié, sur laquelle s'endormait le consultant de l'oracle, qui le mettait en rapport avec Amphiaraos.

En Italie, Latinus s'inquiète en raison de deux prodiges: un essaim suspendu au sommet d'un laurier dans le palais royal et le feu de l'autel domestique embrasant la chevelure de sa fille Lavinia, sa parure, son bandeau royal, sa couronne constellée de gemmes. Son père Faunus est un devin qui rend des oracles « au pied de la haute Albunée, une grande forêt aux vapeurs méphitiques $»^{56}$. Ce n'est pas celle qui avoisine Tibur, mais la «Zolforata», près de Pratica di Mare ${ }^{57}$. Latinus devrait pouvoir communiquer directement avec son père. Mais Faunus est un dieu. Alors, «c'est là quand le

51. Strab., VI, 3, 9 (t. III, p. 185 de l'éd.-trad. F. Lasserre², Paris, Les Belles Lettres ( Collection des Universités de France »), 2003).

52. Alex., 1050-1051. Voir la traduction en partie discutable de Pascal Quignard, Paris, 1971, p. 134.

53. A. Bouché-Leclerq, Histoire de la divination, p. 336, rééd., p. 767.

54. Paus., I, 34, 5.

55. Philostr., Imag., I, 27, 3. La corne nous rappelle Virgile (Aen., VI, 893). Philostrate songe évidemment aux vers de l'Odyssée, XIX, 562-567.

56. Verg., Aen., VII, 83-84.

57. Jérôme Carcopino, Virgile et les origines d'Ostie ${ }^{2}$, Paris, Presses universitaires de France, 1968, p. 300-303. Cf. R. E. A. Palmer, Roman Religion, p. $80-89$. 
prêtre a porté ses offrandes, quand dans la nuit silencieuse il s'est couché sur les peaux étendues des brebis sacrifiées, puis endormi, qu'il entend des voix diverses et jouit de l'entretien des dieux, qu'il parle aux ombres de l'Achéron au fond des enfers $»^{58}$.

Latinus immole rite cent brebis et se couche sur leur toison. Leur nombre est à la mesure royale d'un souverain des Laurentes. Faunus annonce alors à son fils l'avenir de Lavinia et de sa glorieuse descendance.

Plus tard, Numa Pompilius a des obligations similaires, quand les récoltes étant mauvaises, il se rend dans «l'antique forêt... consacrée au dieu du Ménale ${ }^{59}$. Numa immole deux brebis, l'une à Faunus, l'autre à Somnus (le Sommeil). Deux fois, il se baigne la tête à l'eau d'une source pure. Deux fois aussi, il la ceint d'une couronne de hêtre. Il s'est interdit «les plaisirs de Vénus », s'est abstenu de chair animale et ne porte aucun anneau au doigt. En effet, il s'agit pour lui d'éviter toute espèce de lien ou de souillure risquant de s'interposer entre lui et l'influx divin. Ainsi, « couvert d'un vêtement grossier », il s'étend sur les toisons fraîches, avant d'adresser à Faunus les prières appropriées ${ }^{60}$.

Certes, «la Terre est mère des songes $»^{61}$, mais c'est la peau des victimes consacrées qui, en reliant Numa à Faunus, comme Latinus à son père, leur permet d'avoir une révélation expliquant le prodige.

Cette pratique divinatoire se retrouve en Irlande, où les druides dressaient un treillis de sorbier, avant d'y étaler la peau des bœufs sacrifiés, le côté sanglant à l'extérieur, afin d'obtenir des démons, alors forcés à se manifester, la prédiction de l'avenir. Le druide s'étendait sur les dépouilles qu'il rabattait sur lui avec le sang des victimes $^{62}$. Ce faisant, il s'identifiait avec l'animal et entrait dans un rapport étroit avec les dieux.

58. Verg., Aen., VII, 86-91.

59. Ou., F, IV, 649-650. Le Ménale est le mont d'Arcadie où l'on honorait Pan, équivalent de Faunus.

60. Ibid., 654-660. Cf. l'édition commentée de Franz Bömer, Die Fasten, Heidelberg, Carl Winter Universitätsverlag, 1958, p. 265-267. Voir également S. Eitrem, Opferritus und Voropfer, p. 372 ss. ; R. E. A. Palmer, Roman Religion, p. 81-83.

61. H. Lechat, dans DAGL, III, 1, s.u. Incubatio, p. 458.

62. Jan De Vries, La religion des Celtes, trad. franç., Paris, Payot, 1975, p. 239-240. Cf. Thomas Francis O'Rahilly, Early Irish History and Mythology, Dublin, Dublin Institute for Advanced Studies, 1946, p. 324-325. 
Plus récemment, au XVIII ${ }^{\mathrm{e}}$ siècle encore, s'asseoir sur une peau de taureau dans la nuit précédant les Calendes de janvier, à la tête de deux chemins, passait pour aider à prévoir ce qui se passerait l'année suivante ${ }^{63}$. Il n'est pas question alors de sacrifices. Mais souvent une croix exorcisait les carrefours, car dans l'Antiquité Diane, Manium potens Triuia ${ }^{64}$, y régnait sur les esprits des morts et donc les sacralisait, ce qui rendait le lieu propice aux rêveries prophétiques. De toute façon aussi le contact avec la peau animale restait une obligation significative, à laquelle le paganisme populaire pourrait n'être pas tout à fait étranger.

Aux Calendes de janvier, les masques zoomorphes, les pelures et autres dépouilles animales, que les chrétiens ${ }^{65}$ contestaient comme des coutumes païennes, passaient sans doute comme les moyens magico-religieux d'assurer une année bénéfique ${ }^{66}$.

\section{III/ LA DÉIFICATION}

Dans la divinisation iconographique des empereurs romains, les peaux animales occupent une place en image apparemment limitée, mais qui n'en est pas moins explicite. L'égide ${ }^{67}$ et la léonté ${ }^{68}$ les assimilent en image à Zeus ou à Hercule, qui ont libéré le monde des monstres troublant la paix de l'humanité. Les dépouilles ne

63. Pierre Le Brun, Histoire critique des pratiques superstitieuses qui ont séduit les peuples et embarassé les Sçavants..., Rouen, s. n., 1702 ; rééd., 2, Paris, Poirion, 1751, p. 562. Cité par Waldemar Déonna, «Questions d'archéologie religieuse et symbolique, VI », RHR, t. 70, 1914, p. 132, n. de la page précédente.

64. Apul., Apol., 31, 9; Cf. Adam Abt, Die Apologie des Apuleius von Madaura und die antike Zauberei, Giessen, Töpelmann, 1908 («RGVV», 4, 2), rééd., Berlin, Töpelmann, 1967, p. 203-204.

65. Max. Taur., Hom., 16, 4, 46 (PL, 57, col. 257 C) : Cum se a Deo formati homines aut in pecudes, aut in feras, aut in portenta transformant... ; Caesar. Arel., Serm., 13, 5 (éd. trad. Marie-Josée Delage, Paris, Le Cerf ( Sources chrétiennes », 175), p. 139 ; 426, et n. 1 ; Serm., 192, 2 et 193, 2.

66. Cf. Michel Meslin, La fête des kalendes de janvier dans l'empire romain. Étude d'un rituel de nouvel an, Bruxelles, Latomus, 1970 («Latomus », 115), p. $80,82,100-101,122$.

67. Pierre Bastien, Le buste monétaire des empereurs romains, 2, Wetteren, Éditions numismatiques romaines, 1993, p. 341-368 ; pour Gallien, cf. R. Turcan, « Le culte impérial», p. 1040-1041.

68. P. Bastien, loc. cit., p. 369-384 ; A. Alföldi, Die monarchische Repräsentation, p. 239-240 ; R. Turcan, loc. cit., p. 1041. 
sont pas celles de victimes sacrificielles ${ }^{69}$. Mais leur présence sur la poitrine ou les épaules du souverain signifie bien qu'elles le dotent d'un pouvoir «invincible» (il est, de fait, Inuictus), pour assurer l'ordre dans l'Orbis Romanus et le préserver des Barbares. Mais cet aspect relève de l'ordre symbolique dans l'iconographie officielle.

Plus significatif (et problématique) apparaît le port de la nébride accrochée à l'épaule ou ceinte autour des reins. En revêtant la peau d'un bouc immolé à Bacchus, les bacchants ont part à la sacralisation de la victime ${ }^{70}$. Déjà, au IV ${ }^{\mathrm{e}}$ siècle av. J.-C., Démosthène moquait son adversaire Eschine d'avoir participé à des jongleries nocturnes apparentées à des Bacchanales. Il avait « nébrisé », porté la peau de faon ${ }^{71}$. Harpocratiôn ${ }^{72}$ nous explique que le verbe en question nous réfère soit l'ajustement de la peau de faon pour les initiés, soit au démembrement de l'animal suivant un «logos ineffable». Ce logos est celui d'un hiéros logos. D'ailleurs Photius $^{73}$ écrit que le verbe signifie soit porter la nébride, soit démembrer des faons «à l'imitation de la passion de Dionysos » ${ }^{74}$. Cette «passion» est celle de Dionysos démembré par les Titans. En réalité, les deux exégèses que nous livrent les lexicographes sont solidaires. Les bacchants portent la nébride, parce qu'ils s'identifient avec leur dieu souffrant.

On connaît la suite. Zeus foudroie les Titans criminels et le sublimé des vapeurs qui s'élèvent alors au-dessus d'eux sert à créer les

69. Rappelons pourtant que le premier des travaux d'Hercule compte au nombre des expiations auxquelles le héros dut se soumettre après le meurtre de ses enfants (Pierre Chuvin, La mythologie grecque. Du premier homme à l'apothéose d'Héraclès, Paris, Fayard, 1992, p. 194). Notons également que le mythe était associé aux rituels du sacrifice accompli lors des Jeux Néméens (ibid., p. 207).

70. Robert Turcan, Liturgies de l'initiation bacchique à l'époque romaine. Documentation littéraire, inscrite et figurée, Paris, De Boccard, 2003 (« Mémoires de l'Académie des Inscriptions et Belles-Lettres », 27), p. 16 (44).

71. Dem., Cor., 259. Cf. Amb., 199 («thiases d'ivrognes »). Cf. Henri Jeanmaire, Dionysos. Histoire du culte de Bacchus, Paris, Payot, 1951, p. 94-97.

72. Lex., s.u. nebrizôn, I, p. 212, 15 ss. Dindorf.

73. Lex., s.u., I, p. 441 Naber.

74. Même donnée dans Lex. Patm. (Kurt Latte - Hartmut Erbse, Lexica Graeca minora, réimpr., Hildesheim, Olms, 1992, p. 154) et dans la Souda, qui transcrit l'explication d'Harpocratiôn. Voir aussi H. Jeanmaire, Dionysos, p. 253-254 ; R. Turcan, Liturgies de l'initiation bacchique, p. 16, 42. Sur le mythe : Alberto Bernabé, «La toile de Pénélope : a-t-il existé un mythe orphique sur Dionysos et les Titans ?», RHR, t. 219, 2002, p. 401-433. 
hommes ${ }^{75}$. Ils ont donc une double hérédité, titanique et dionysiaque. C'est ainsi qu'en revêtant la peau du faon ou du chevreau sacrifié, les bacchants s'identifient avec leur dieu. Leur initiation en fait des $\mathrm{Bacchoi}^{76}$, surtout s'ils ont dévoré la chair crue de l'animal qui pouvait être une chèvre noire ${ }^{77}$, ou un taureau ${ }^{78}$. La tradition littéraire et les monuments figurés illustrent cet aspect sanglant de l'orgiasme ${ }^{79}$.

On songe évidemment aussi à une tablette de Thourioi, où la reine des Enfers dit à l'initié « Ô bienheureux ! Tu es devenu dieu d'homme que tu étais », et l'initié répond: «chevreau, je suis tombé dans le lait ${ }^{80}$. Clément d'Alexandrie ${ }^{81}$ nous donne l'explication : «Les Titans qui l'avaient dépecé, plaçant une marmite sur un trépied y jetèrent ses membres. Après les avoir bien fait bouillir, ils les transpercèrent avec des brochettes et les tinrent "au-dessus d'Héphaistos" (Hom., Il., II, 426) » : autrement dit, « sur le feu ». C'est dans le lait que les Titans font d'abord bouillir Dionysos, avant de le faire griller ${ }^{82}$.

75. Otto Kern, Orphicorum fragmenta ${ }^{3}$, Dublin - Zurich, Weidmann, 1972, p. 238, $\mathrm{n}^{\mathrm{o}} 220$ =Olympiod., In Plat., Phaedon., 61 c, p. 2, 211 ss. Norvin. Cf. Luc Brisson, "Le corps "dionysiaque" ", Sophiès Maietores "chercheurs de sagesse ». Hommage à Jean Pépin, éd. Odile Goulé-Casé, Goulven Madec, Denis O’Brien, Paris, Institut d'études augustiniennes, 1992 ( Collection d'études augustiniennes », 131), p. 481-499.

76. Schol. Aristoph., Eq., 408 (p. 103, 8-9 Jones). Les mystes d'Hipponion sont des Bacckoi: Giovanni Pugliese Carratelli, Les lamelles d'or orphiques. Instructions pour le voyage d'outre-tombe des initiés grecs, trad. franç., Paris, Les Belles Lettres, 2003, p. 34, ligne 15. Cf. le chœur des Crétois, dans François Jouan et Herman Van Looy, éd. et trad. Euripide, VIII, 2, Fragments, Paris, Les Belles Lettres, 2000 ( «Collection des Universités de France»), p. 324, 2 (= 471 Kannicht) : «Ayant accompli les rites de l'omphagie... j'ai été consacré et reçus le nom de "Bacchos"... ». Cf. aussi G. Pugliese Carratelli, op. cit., p. 90-91.

77. R. Turcan, Liturgies de l'initation bacchique, p. 16, 44.

78. Ibid., p. 40-41, 96. Cf. André Boulanger, Orphée, rapports de l'orphisme et du christianisme, Paris, Rieder, 1925, p. 37 : «l'initié qui a consommé la chair crue de l'animal représentant le dieu est possédé par Dionysos, et mieux, devient lui-même un "bacchos"..." .

79. H. Jeanmaire, Dionysos, p. 251-257 ; Robert Turcan, Les sarcophages romains à représentations dionysiaques. Essai de chronologie et d'histoire religieuse, Paris, De Boccard, 1966 («Bibliothèque des Écoles françaises d'Athènes et de Rome », 210), p. 205, 290, 435, 495, 538-541.

80. O. Kern, Orphicorum fragmenta ${ }^{2}$, p. 106-107, no 32 c, 10-11. Cf. Louis Moulinier, Orphée et l'orphisme à l'époque classique, Paris, Les Belles Lettres, 1955 , p. 55 : « Le chevreau est Bacchos ».

81. Protr., II, $18,1=$ O. Kern, Orphicorum fragmenta ${ }^{2}$, p. $111, \mathrm{n}^{\circ} 35$.

82. Cf. William K. C. Guthrie, Orphée et la religion grecque, trad. franç., Paris, Payot, 1956, p. 199-200 et 232-233. 
Quoi qu'il en soit, la nébride paraît bien correspondre à un rituel de déification. Il est troublant de constater qu'aux Calendes de janvier Césaire d'Arles déplore encore (au début du VI siècle) les déguisements $"$ en agnelle ou en faon $»^{83}$. Rien n'interdit de penser qu'il s'agissait d'animaux immolés clandestinement.

Sur les déguisements zoomorphes dans les mystères de Mithra, nous sommes assez peu ou plutôt mal informés ${ }^{84}$. Il est douteux que les mystes aient toujours revêtu les dépouilles d'animaux sacrifiés. Mais il est notable aussi que Mithra partage souvent avec Sol un repas d'alliance - acte sacré par excellence - sur la peau du taureau ${ }^{85}$.

La dépouille d'un animal sacrifié a des vertus multiples, d'ordre surnaturel, qu'elles soient cathartiques, prophétiques ou déifiques, car elle renferme en elle « une partie de la vie divine $»^{86}$.

turcan.isabelle@orange.fr

83. Serm., 13, 5 (éd et trad. M.-J. Delage, p. 426 et n. 1).

84. Franz Cumont, Les mystères de Mithra ${ }^{4}$, Savigliano, Aragno, 2013, p. 121 : ces travestissements « remontent à cette période de l'histoire ou de la préhistoire où l'on se représentait les divinités elles-mêmes sous une forme animale, et où le fidèle, en prenant le nom ou l'aspect de son dieu, croyait s'identifier avec lui ». Mais nos sources restent discutables, sinon parfois erronées: Robert Turcan, «Une aporie de la tradition littéraire sur le "Lion" mithriaque», Les religions orientales dans le monde grec et romain: cent ans après Cumont (1906-2006). Bilan historique et historiographique, éd. Corinne Bonnet, Vinciane PirenneDelforge, Danny Praet, Bruxelles - Rome, Institut historique belge de Rome, 2006 («Études de philologie, d'archéologie et d'histoire anciennes », 45), p. 429-448.

85. Robert Turcan, Mithra et le mithriacisme ${ }^{4}$, Paris, Les Belles Lettres, 2004, p. $57,79,98,102$.

86. J. G. Frazer, Le rameau d'or, p. 472. 